\title{
Insight change in psychosis: relationship with neurocognition, social cognition, clinical symptoms and phase of illness
}

Quee PJ, van der Meer L, Krabbendam L, de Haan L, Cahn W, Wiersma D, van Beveren N, Pijnenborg GHM, Mulder CL, Bruggeman R, Aleman A. Insight change in psychosis: relationship with neurocognition, social cognition, clinical symptoms and phase of illness.

Objective: Impaired insight is an important and prevalent symptom of psychosis. It remains unclear whether cognitive disturbances hamper improvements in insight. We investigated the neurocognitive, social cognitive, and clinical correlates of changes in insight.

Method: One hundred and fifty-four patients with a psychotic disorder were assessed at baseline $\left(T_{0}\right)$ and after three years $\left(T_{3}\right)$ with the Birchwood Insight Scale, the Positive And Negative Syndrome Scale, measures of neurocognition and social cognition. Linear regression analyses were conducted to examine to what extend neurocognition, social cognition, clinical symptoms and phase of illness could uniquely predict insight change. Subsequently, changes in these factors were related to insight change.

Results: Better neurocognitive performance and fewer clinical symptoms at baseline explained insight improvements. The additional effect of clinical symptoms over and above the contribution of neurocognition was significant. Together, these factors explained $10 \%$ of the variance. Social cognition and phase of illness could not predict insight change. Changes in clinical symptoms, but not changes in neurocognitive performance were associated with insight change.

Conclusion: Neurocognitive abilities may predict, in part, the development of insight in psychosis.

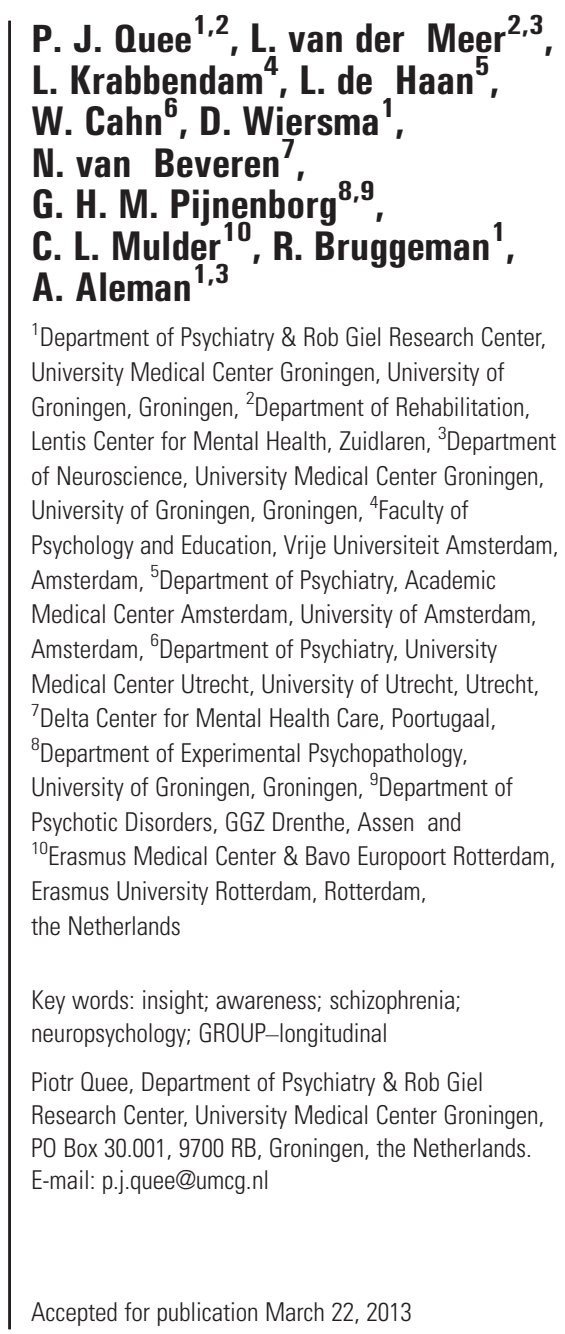

\section{Significant outcomes}

- In a longitudinal design, neurocognition and clinical symptoms at baseline were uniquely related to insight change after 3 years.

- Changes in clinical symptoms, but not changes in neurocognitive performance, were related to insight change.

- Social cognition and phase of illness were not related to insight change.

\section{Limitations}

- A substantial subgroup did not participate in the study at follow-up.

- The assessment of social cognition and insight was limited. 


\section{Introduction}

Impaired insight, or unawareness of illness, is a highly prevalent symptom of patients with a psychotic disorder (1). Insight can be studied as a set of descriptive beliefs and as a personal narrative (2). In the current study, insight was studied as a set of descriptive beliefs, including the following aspects: i) awareness of illness, (ii) need for treatment and (iii) relabelling of symptoms (3). Impaired insight has adverse consequences on outcomes of the disease, including functional outcome, treatment adherence and re-admissions (4-6). Multiple factors have been associated with reduced insight, including neurocognitive impairment, social cognition and clinical symptoms (7, 8). However, the majority of the studies investigating insight applied a cross-sectional design, leaving the question whether the above factors contribute to the course of insight over time unanswered. This may be particularly relevant information for clinicians to be able to develop treatment strategies to improve insight.

Of the neurocognitive domains, executive impairment (working memory, reasoning/problem solving) seems to contribute mostly to poor insight in psychotic disorders, although in schizophrenia, impairments in multiple domains of neurocognition (i.e. verbal learning and memory, attention/ vigilance) have been related to insight (7). Only a few studies have investigated the course of insight longitudinally. Parellada et al. demonstrated that cognitive impairments predicted several aspects of insight after two years (9). However, their assessment did not include all of the neurocognitive domains known to be impaired in schizophrenia (10). A second study failed to find a significant effect of neurocognition on future insight (11). In a third study, patients with improved insight were found to have better cognitive performance after six months on some tasks, but not all (12). However, these findings may have been the result of their inclusion of medication-naive patients with a first psychotic episode at baseline. Therefore, although it has been suggested that cognitive impairments limit the response to psychosocial treatment in general (13), it has not yet been addressed adequately whether this applies to insight improvement as well.

Previously, we demonstrated that social cognition and clinical symptoms are both uniquely related to level of insight, irrespective of neurocognitive functioning; interestingly, phase of illness moderated this effect (14). Taking these findings into account, it is interesting to study whether neurocognition, social cognition, clinical symptoms and phase of illness are also predictive of changes in insight. In addition, it is not known whether changes in these factors fluctuate with changes in insight change. Increases in severity of clinical symptoms have been found to be associated with decreases in insight, but only in patients with recent onset psychosis (15).

\section{Aims of the study}

In the current study, we investigated factors associated with change in insight over time in a longitudinal design. We expected that neurocognition and social cognition would explain changes in insight over time. We also investigated whether changes in neurocognition, social cognition, clinical symptoms and phase of illness were associated with changes in insight.

\section{Material and methods}

Participants

Two hundred and seventy patients with psychotic disorders (predominantly schizophrenia) were assessed with the Birchwood Insight Scale at baseline (see Assessments). This was a subsample of the patient population participating in the GROUP project (16). The GROUP project is a large-scale multicentre study that investigates the vulnerability and protective factors for i) the development of a psychotic disorder and ii) the variation of the course of illness. Two of four centres participated in the insight study (Amsterdam and Utrecht). Diagnoses were confirmed using the Comprehensive Assessment of Symptoms and History (17). To maximize uniformity in experimental procedures and experimenter behaviour, all interviewers received extensive training. Interviewers were research assistants, psychologists, psychiatrists, nurses and PhD students. The procedure of recruitment, informed consent, assessment instruments, assessment training, approval by an accredited Medical Ethics Review Committee and population characteristics have been described in a previous report on the project (13).

\section{Assessments}

Insight. Insight was assessed using two measures. From the Positive And Negative Syndrome Scale (PANSS) (18), a semistructured interview consisting of 30 items, we used item G12 (Poor Judgement and Insight). Scores on the PANSS range from 1 (absent) to 7 (extremely severe). Secondly, we used the Birchwood Insight Scale (BIS) (19). 


\section{Quee et al.}

The BIS is a short self-rating questionnaire that consists of eight questions addressing the three components of insight in psychosis (Awareness of Illness, Need for Treatment and Relabeling of Symptoms). The BIS total score ranges from 0 to 12 , with higher scores indicating better insight. For insight, as well as neurocognition, social cognition and clinical symptoms, composite measures were created (see Statistical analysis). For insight, the composite measure consisted of the PANSS item G12, and the BIS.

Neurocognition. The neurocognitive measures used in the GROUP study have been described in detail elsewhere (20). The following tasks were administered: Continuous Performance Test-HQ (CPTHQ) (attention/vigilance), Response Set-shifting Task (RST) (reasoning and problem solving), short form of the Wechsler Adult Intelligence Scale-III (WAIS-III) containing the subtests Block Design (reasoning and problem solving), Digit Symbol (processing speed), Arithmetic (working memory) and Information (verbal comprehension) (21), Word Learning Task (WLT) immediate recall and delayed recall (verbal learning and memory) (22). Educational degree was assessed using nine categories, zero being the lowest (no education) and eight being the highest (academic degree) (23). The parameters used for neurocognition were similar as in our previous study (14), with a few exceptions. For the CPT-HQ, two parameters were created: CPT variance and CPT performance index. For CPT variance, or intraindividual variability (24), the standard deviation of the subject's mean response time on the hit trials was used. CPT performance index consisted of an efficiency score [(accuracy/reaction time $) \times 1000]$, in which accuracy was measured as the total number of hits (range, 0-28) minus the total number of errors (range, 0-28), divided by 28. The RST parameter was based on the subjects' accuracy during the experimental condition, during which the subject has to find the alternated response rule. For all neurocognitive tests, higher scores indicated better performances, except for CPT variance.

Social Cognition. For social cognition, the Degraded Facial Affect Recognition task (emotion perception) (25) and the Hinting Task (Theory of Mind) (26) were used. Higher scores on the measures of social cognition reflected better performances. The Hinting Task was measured at baseline only.

Clinical Symptoms. Current symptom severity was measured with the PANSS. Items on the PANSS incorporate the behavioural effect of the symptoms, as well as their severity. Interrater reliability, evaluated with ICC, was found to be high for the positive subscale, the negative subscale and total PANSS score $(0.957,0.911$ and 0.946 , respectively) (16).

As in our earlier study (14), we calculated a mean score of the subject's ratings on the eight remission items (27). These items include: delusions (P1), conceptual disorganization (P2), hallucinatory behaviour (P3), blunted affect (N1), social withdrawal (N4), and lack of spontaneity (N6), mannerisms/posturing (G5) and unusual thought content (G9).

Phase of Illness. Phase of illness was divided into recent onset psychosis and multiple or chronic psychosis. Recent onset psychosis was defined as follows: a first psychotic episode during the year prior to the baseline assessment. All other patients were characterized as having 'multiple episode or chronic psychosis'.

\section{Statistical Analysis}

Normality was checked for all variables. If necessary, variables were transformed to approximate normality using logarithmic or square root transformation. Subsequently, a composite measure was created for the insight measures at baseline and follow-up. As in our previous study, the scores on the G12 and BIS were transformed into $z$-scores, based on the mean and standard deviation of the baseline assessment (14). Insight change was computed by subtracting the insight composite score at baseline from the insight composite score at follow-up. Thus, higher scores at insight change reflect more insight improvement. A paired $t$-test was used to investigate differences between baseline and follow-up insight.

Next, composite scores were created for baseline neurocognition, cognition and clinical symptoms. The composite score included all test scores of each of the abovementioned factors (see Method and materials: Assessments). For neurocognition and social cognition, this was carried out by transforming all the raw scores into $z$-scores, using the mean and standard deviations from the patient population included at baseline $(n=270)$. For neurocognition, the composite score consisted of eight measures: CPTHQ (average performance index and variance, the latter being negatively transformed), RST, WAIS-III Digit Symbol, Block Design, Arithmetic, and Information, WLT (average immediate and delayed recall) and educational degree. For 
clinical symptoms, a mean score was created, based on the eight PANSS items.

Hierarchical regression analyses investigated the predictive value of neurocognition, social cognition, clinical symptoms and phase of illness at baseline. These variables were entered block-wise, which enabled us to investigate the explained variance of neurocognition, as well as the additional explained variance of social cognition, clinical symptoms and phase of illness. Age at baseline and sex were entered in the first block, as covariates. Scatter plots were used to evaluate the direction of the effects. Changes $\geq 1$ SD were considered insight improvement, whereas changes $\leq-1 \mathrm{SD}$ were considered insight decrease.

Next, we analysed whether changes in neurocognition, social cognition, clinical symptoms and phase of illness were related to insight change. This was carried out by subtracting the score at baseline from the score at follow-up. These change scores were then transformed into $z$-scores to create a composite measure for neurocognition. Thus, for neurocognition change as well as social cognition, higher change scores indicated improved performance; for clinical symptoms change, higher scores indicated more symptoms. Variables were entered block-wise in a new regression analysis, again with the covariates age and sex in the first block.

All analyses were performed with two-tailed hypothesis testing, with $\alpha=0.05$. For the evaluation of the scaled scores and composite measures, we allowed for $30 \%$ of missing values. Statistical analyses were performed using PASW 18.0 (PASW Statistics for Windows, Version 18.0. Chicago, IL, USA: SPSS Inc., 2009). Release 3.0 of the GROUP database was used for the analyses. For descriptive purposes, correlations between all variables are displayed in Table $\mathrm{S} 1$.

\section{Results}

\section{Descriptives}

Of the 270 patients assessed with the assessment of insight at baseline, 154 were assessed in GROUP at follow-up. Drop-out in Utrecht and Amsterdam was relatively similar $(45 \%$ and $55 \%$, respectively). These patients $(n=116)$ received a questionnaire to investigate reasons for drop-out; however, none of these patients responded to this request. Insight at baseline of the remaining patients differed significantly from the drop-out patients $[F(1,272)=12.649, P<0.001]$, with the former having higher levels of insight $(z=0.16$, $z=-0.19$, respectively). The effect size of this dif- ference was small $(d=0.46)$, suggesting that the sample is still relatively reliable. Differences were also significant for education $[F(1,272)=7.605$, $P=0.006]$. The remaining patient group was higher educated, as compared to the drop-out patients. Differences were not significant for neurocognition, social cognition, clinical symptoms, phase of illness, age and sex. Table 1 shows the demographic and clinical data for the included 154 patients of the current study. At baseline, impaired insight defined as PANSS G12 $\geq 3$ ( $3=$ threshold for the presence of a symptom) was found in $45 \%$ of the included patients. On the BIS, $56 \%$ of the patients had a score $\leq 9$ $(9=$ threshold for the presence of insight impairment). At baseline, $21 \%$ of the patients included had a recent onset psychosis.

\section{Insight change: relationship with baseline insight}

The difference between insight at baseline and follow-up was not statistically significant $(P=0.388)$. The relationship between insight at baseline and insight at follow-up was significant $(r=0.415$, $P<0.001)$. Furthermore, baseline insight was significantly related to insight change $(r=0.515$,

Table 1. Baseline characteristics of the patients $(N=154)^{*}$

\begin{tabular}{lcc}
\hline & \multicolumn{2}{c}{ Assessment } \\
\cline { 2 - 3 } Variable & Baseline $\left(T_{0}\right.$ years $)$ & Follow-up ( $T_{3}$ years $)$ \\
\hline Age, years & $28(7)$ & $31(7)$ \\
Gender, male \% & 80 & 80 \\
Education† & $4.6(2.1)$ & $4.9(1.9)$ \\
Duration of illness, years & $5(5)$ & $7(5)$ \\
Diagnostic & & \\
Schizophrenia, \% & 67 & 66 \\
Schizo-affective, \% & 19 & 18 \\
Psychosis NOS, \% & 8 & 5 \\
Other, \% & 6 & 11 \\
Anti-psychotics & & \\
Olanzapine, \% & 32 & 20 \\
Risperidone, \% & 25 & 15 \\
Clozapine, \% & 10 & 20 \\
Aripiprazol, \% & 8 & 16 \\
Ouetiapine, \% & 7 & 9 \\
Other, \% & 11 & 11 \\
No anti-psychotics, \% & 7 & 9 \\
PANSS & & \\
Positive & $1.9(0.8)$ & $1.6(0.6)$ \\
Negative & $2.2(0.8)$ & $1.7(0.7)$ \\
General & $1.8(0.5)$ & $1.5(0.4)$ \\
Insight & & $1.7(1.2)$ \\
G12 & $2.0(1.3)$ & $8.9(2.7)$ \\
BIS & $9.3(2.5)$ & \\
\hline
\end{tabular}

${ }^{*}$ Table presents means (SD) or numbers (in \%).

$\uparrow$ Education (Verhage): range 0 (no education), $3-5$ (school diploma) to 8 (academic degree).

PANSS, Positive And Negative Syndrome Scale; G12, PANSS item G12 'Judgment and Insight'; BIS, Birchwood Insight Scale. 


\section{Quee et al.}

$P<0.001$ ), with better insight at baseline being associated with more insight improvement.

Insight change: relationships with baseline neurocognition, social cognition, clinical symptoms and phase of illness

Insight change was significantly related to baseline neurocognition $(r=0.231, P=0.005)$ and clinical symptoms $(r=-0.215, P=0.009)$. Better baseline neurocognitive performances were related to improvements in insight after 3 years (Fig. 1). Patients with more symptoms at baseline had decreased insight (Fig. 2). The relationship with social cognition and phase of illness was non-significant. When neurocognition and clinical symptoms were entered in the regression analysis consecutively, with age and sex as covariates, the additional explained variance of clinical symptoms was significant. Together, these factors explained insight improvement for $10 \%$ (Table 2).

Insight change: relationships with changes in neurocognition, social cognition and clinical symptoms

The difference between neurocognition at baseline and follow-up was statistically significant $(t=-5.941, P<0.001)$. This was also true for clinical symptoms $(t=6.138, P<0.001)$, but not for social cognition $(P=0.155)$. Better neurocognitive performance and less severe clinical symptoms were found at follow-up. Effect sizes were small for neurocognition $(d=0.25)$ and in the medium range for clinical symptoms $(d=0.51)$. Insight change was significantly related to change in clinical symptoms $(r=-0.223, P=0.008)$. Decreases in clinical symptoms over time were related to increases in insight. The relationships with change in neurocognition, social cognition and phase of illness were non-significant. When change in clinical symptoms was entered in the regression analysis, with age and sex as covariates, the explained variance of the model was $12 \%$, with the contribution of clinical symptoms being significant $(\beta=-0.324, \quad P<0.001)$ (Table 3).

\section{Discussion}

The current study investigated factors associated with insight change in non-affective psychosis. Results can be summarized as follows. Most importantly, we found that, although the mean group-level of insight was relatively stable over time, improvement of insight within patients was positively related to baseline neurocognitive performances and negatively related to baseline symptom severity. In addition, changes in insight were found to fluctuate with changes in symptom severity, but not with changes in neurocognition, between baseline and follow-up.

The course of insight was stable in the majority of this relatively young population. This is in line with findings that interventions to date had only limited success in improving insight over time (28). It therefore becomes relevant to study the underlying factors of this insight stability. Longitudinal studies focusing on insight have provided mixed results $(9,11,12)$. Rather than predicting the future level of insight, our study focused on factors contributing to changes in insight. The advantage of this approach is that using a difference score as the outcome measure, both the level of insight at

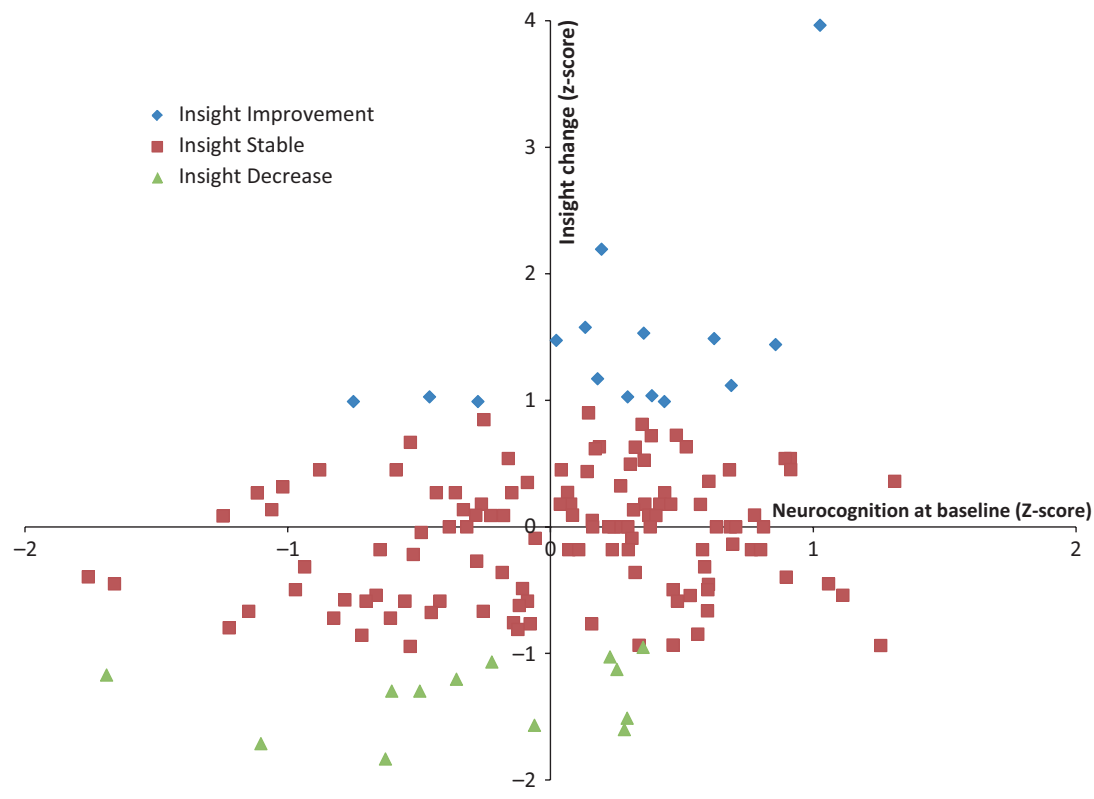

Fig. 1. Insight change as a function of baseline neurocognition. 
Fig. 2. Insight change as a function of baseline clinical symptoms.

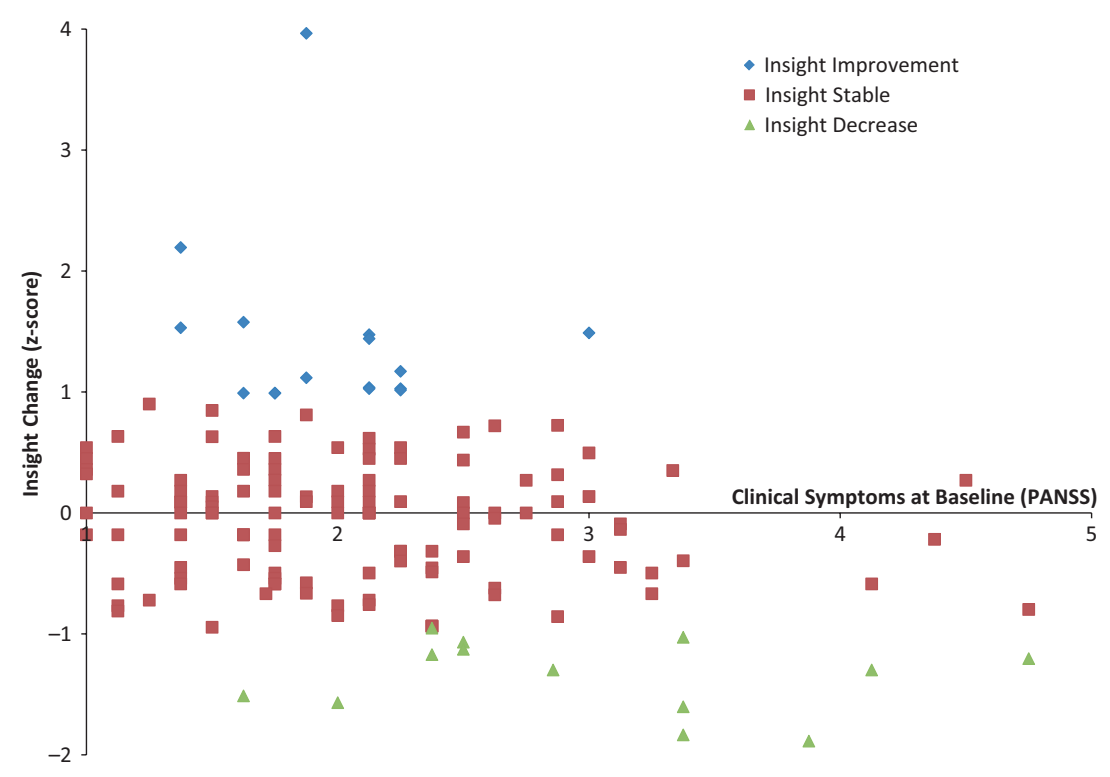

baseline, as well as insight at follow-up are taken into account.

In line with the hypotheses, baseline neurocognition substantially contributed to insight change, together with clinical symptoms. This shows that neurocognitive impairments may have a limiting effect on insight. Baseline symptom severity as well as decreases in symptoms over time was associated with an increase in insight as well. This may be explained by the fact that the patients in our sample improved in terms of clinical symptoms $(d=0.51)$. Neurocognitive improvement, on the other hand, was only small $(d=0.25)$. Neurocognition may thereby contribute to 'trait' characteristics of insight, whereas clinical symptoms contribute to 'state' characteristics. Possibly, the current treatments for psychosis are already partly beneficial to improve state characteristics of insight in psychosis. In a subsequent analysis, we found that some of the neurocognitive abilities and clinical symptoms may be particularly responsible for this (Table S1). The relationship between these significant variables should be tested in a new sample to confirm their predictive value on insight change.
In contrast to our expectations, phase of illness and social cognition were not related to insight change. Therefore, insight change may not differ between recent onset patients and other patients. On the other hand, it cannot be ruled out that the correlates of insight change would differ in a study covering a longer period of time. In an earlier study, we found social cognition to be of additional value for insight as well (14). Social cognition has been found to mediate the relationship between neurocognition and functional outcome (29). Neurocognitive impairments may thereby underlie the deficits in emotion perception and

Table 3. Relationships of changes in clinical symptoms with insight change for patients with psychotic disorders

\begin{tabular}{lcccccc}
\hline & \multicolumn{5}{c}{ Insight change $\left(T_{0}\right.$ years $-T_{3}$ years $)$} \\
\cline { 2 - 7 } Model & df & $\begin{array}{c}\beta_{\text {Clinical }} \\
\text { symptoms }\end{array}$ & $P$ & $F$ & $R$ & $R^{2}$ \\
\hline $\begin{array}{l}\text { Change in clinical } \\
\text { symptoms }\end{array}$ & 3,138 & $-0.343 \dagger$ & 0.001 & 6.087 & 0.342 & 0.117 \\
\hline
\end{tabular}

$\beta=$ standardized beta coefficient.

†Correlation significant at the 0.01 level. Included covariates are age and sex.

Table 2. Relationships of baseline neurocognition and clinical symptoms with insight change for patients with psychotic disorders

\begin{tabular}{lccccccccccc}
\hline & \multicolumn{10}{c}{ Insight change $\left(T_{0 \text { years }}-T_{3}\right.$ years } & \\
\cline { 2 - 10 } Model & $\mathrm{df}$ & $\beta_{\text {Cognition }}$ & $\beta_{\text {clinical symptoms }}$ & $P$ & $F$ & $R$ & $R^{2}$ & $P_{\text {Change }}$ & $F_{\text {Change }}$ & $R_{\text {Change }}^{2}$ \\
\hline Neurocognition & 3,137 & $0.259^{*}$ & - & 0.018 & 3.450 & 0.265 & 0.070 & - & - \\
+ Clinical symptoms & 4,136 & $0.200 \dagger$ & $-0.176 \dagger$ & 0.007 & 3.660 & 0.312 & 0.097 & 0.046 & 4.061 & 0.027 \\
\hline
\end{tabular}

$\beta=$ standardized beta coefficient; $P_{\text {change, }} F_{\text {change, }}, R_{\text {change, }}$ and $R_{\text {change }}^{2}$ refer to the statistical significance of the model as compared with its preceding model.

${ }^{*}$ Correlation significant at the 0.01 level. Included covariates are age and sex.

$\dagger$ Correlation significant at the 0.05 level. 


\section{Quee et al.}

theory of mind, and this may also apply to the enduring impairments in insight.

The current study may have clinical implications. It has been suggested that patients with more severe neurocognitive impairments are less able to profit from psychosocial interventions (30). This may also explain why they only minimally improve insight. Such interventions may need to be provided with a high level of structure, and more rehearsal, to improve insight. We did not find a relationship between neurocognitive change and insight change. Still, this does not rule out a role of cognitive remediation here. Enhanced cognition, together with decreased symptoms may give the patients more possibilities to increase their awareness of illness over time.

Strengths of the current study were its longitudinal design, the assessment of several cognitive domains and the methodology used. Some limitations should also be considered. First, a substantial subgroup did not participate in the GROUP study at follow-up. Although effect sizes were small, patients who did not participate at follow-up study had poorer insight at baseline. Reasons for drop-out were unknown. Second, our assessment of social cognition was limited. We did not include a measure of metacognition, which may be more closely related to insight (31). Third, stigma and depressive symptoms were not assessed in the current study (3234). Fourth, the assessment of insight may have been more comprehensive would we have used the Structured Assessment of Insight-Expanded (34). It cannot be ruled out that this would had changed the level of insight at baseline, and the contribution of social cognition to insight change which was non-significant in the current study. Due to these limitations our results should be interpreted cautiously.

In conclusion, the current study highlights the role of neurocognition and clinical symptoms for insight improvement. Therefore, it may be necessary to develop treatment strategies that incorporate these aspects, among others such as stigma, metacognition and depressive symptoms (35).

\section{Acknowledgements}

The GROUP study was supported by the Geestkracht programme of the Dutch Health Research Council (ZON-MW, grant number 10-000-1002). The authors would like to thank René Kahn, Don Linszen, Jim van Os, Inez Germeys, Carin Meijer, Philippe Delespaul, Peter de Jonge, Dick Smid, Nikie Korver, Agna Bartels, Heleen Boos, Joyce van Baaren, Leo Swart, Erwin Veermans, Truda Driesen, all other involved staff members participating in the GROUP project and all participants for their time and cooperation.

\section{Declaration of interest}

None.

\section{References}

1. Amador XF, Gorman JM. Psychopathologic domains and insight in schizophrenia. Psychiatr Clin North Am 1998; 21:27-42

2. Roe D, Kravetz S. Different ways of being aware of psychiatric disability: a multifunctional narrative approach to insight into mental disorder. J Nerv Ment Dis 2003; 191:417-424

3. DAVID AS. Insight and psychosis. Br J Psychiatry 1990; 156:798-808.

4. Jónsdóttir H, Opjordsmoen S, Birkenaes AB et al. Predictors of medication adherence in patients with schizophrenia or bipolar disorder. Acta Psychiatr Scand 2013; 127:23-33.

5. Ruissen AM, Widdershoven GA, Meynen G, Abma TA, van BALKOM AJ. A systematic review of the literature about competence and poor insight. Acta Psychiatr Scand 2012;125:103-113.

6. Drake RJ, Dunn G, Tarrier N, Bentall RP, Haddock G, LEwIS SW. Insight as a predictor of the outcome in firstepisode nonaffective psychosis in a prospective cohort study in England. J Clin Psychiatry 2007;68:81-86.

7. Aleman A, Agrawal N, Morgan KD, David AS. Insight in psychosis and neuropsychological function: a metaanalysis. Br J Psychiatry 2006;189:204-212.

8. Mintz AR, Dobson KS, Romney DM. Insight in schizophrenia: a meta-analysis. Schizophr Res 2003;61:75-88.

9. Parellada M, Boada L, Fraquas D et al. Trait and state attributes of insight in first episodes of early-onset schizophrenia and other psychoses: a 2-year longitudinal study. Schizophr Bull 2011;37:38-51

10. Nuechterlein KH, Barch DM, Gold JM, Goldberg TE, Green MF, Heaton RK. Identification of separable cognitivefactors in schizophrenia. Schizophr Res 2004;72: 29-39.

11. Cuesta Mu, Peralta V, Zarzuela A, Zandio M. Insight dimensions and cognitive function in psychosis: a longitudinal study. BMC Psychiatry 2006;31:26.

12. Cuesta MJ, Peralta V, Compos MS, Garcia-Jalon E. Can insight be predicted in first-episode psychosis patients? A longitudinal and hierarchical analysis of predictors in a drug-naïve sample. Schizophr Res 2011;130:148-156.

13. Lysaker PH, Clements CA, Plascak-Hallberg CD, KNIPSCHEER SJ, WRIGHT DE. Insight and personal narratives of illness in schizophrenia. Psychiatry 2002;65:197-206.

14. Quee PJ, van der Meer L, Bruggeman R et al. Insight in psychosis: relationship with neurocognition, social cognition and clinical symptoms depends on phase of illness. Schizophr Bull 2011;37:29-37.

15. Buchy L, Bodnar M, Malla A, Joober R, Lepage $M$. A 12-month outcome study of insight and symptom change in first-episode psychosis. Early Interv Psychiatry 2012;4:79-88.

16. Korver N, Quee PJ, Boos HB, Simons CJ, De Haan L, Group Investigators. Genetic Risk and Outcome of Psychosis (GROUP), a multi site longitudinal cohort study focused on gene-environment interaction: objectives, sample characteristics, recruitment and assessment methods. Int J Methods Psychiatr Res 2012;21:205-221.

17. Andreasen NC, Flaum MC, Arndt S. The Comprehensive Assessment of Symptoms and History (CASH): an instru- 


\section{Insight change in psychosis}

ment for assessing diagnosis and psychopathology. Arch Gen Psychiatr 1992;49:615-623.

18. Kay SR, Flszbein A, Opfer LA. The positive and negative syndrome scale (PANSS) for Schizophrenia. Schizophr Bull 1987;13:261-276.

19. Birchwood M, Smith J, Drury V, Healy J. A self-report insight scale for psychosis: reliability, validity and sensitivity to change. Acta Psychiatr Scand 1994;89: 62-67.

20. Meijer J, Simons CJP, Quee PJ, Verweis K, Group Investigators. Cognitive alterations in patients with non-affective psychotic disorder and their unaffected siblings and parents. Acta Psychiatr Scan 2011;125:66-76.

21. Blyler CR, Gold JM, Iannone VN, Buchanan RW. Short form of the WAIS-III for use with patients with schizophrenia. Schizophr Res 2000;46:209-215.

22. Brand N, Jolles J. Learning and retrieval rate of words presented auditorily and visually. J Gen Psychol 1985; 112:201-210.

23. Verhage F. Intelligentie en leeftijd: onderzoek bij Nederlanders van twaalf tot zevenenzeventig jaar. Assen: Van Gorcum, 1964.

24. Hilti CC, Hilti LM, Heinemann D, Robbins T, Seifritz E, Cattapan-Ludewig K. Impaired performance on the Rapid Visual Information Processing task (RVIP) could be an endophenotype of schizophrenia. Psychiatr Res 2010; 177:60-64

25. Van 'T Wout M, Alemana A, Kessels RPC, Larøi F, KahN RS. Emotional processing in a non-clinical psychosisprone sample. Schizophr Res 2004;68:271-281.

26. Corcoran R, Mercer G, Frith CD. Schizophrenia, symptomatology and social influence: investigating theory of mind' in people with schizophrenia. Schizophr Res 1995; 17:5-13.

27. Andreasen NC, Carpenter WT JR, Kane JM, Lasser RA, Marder SR, Weinberger DR. Remission in Schizophrenia: proposed criteria and rationale for consensus. Am J Psychiatry 2005;162:441-449.

28. Pijnenborg GHM, Donkersgoed R, David AT, Aleman A. Changes in insight during treatment for psychotic disorders: a meta-analysis. Schizophr Res 2012;144:109117.

29. Schmidt SJ, Mueller DR, Roder V. Social cognition as a mediator variable between neurocognition and functional outcome in schizophrenia: empirical review and new results by structural equation modeling. Schizophr Bull 2011;37(Suppl. 2):S41-S54.

30. LySAKER PH, Buck KD. Neurocognitive deficits as a barrier to psychosocial function in schizophrenia: effects on learning, coping, \& self-concept. J Psychosoc Nurs Ment Health Serv 2007; 45:24-30.

31. Koren D, Seidman LJ, Poyurovsky M et al. The neuropsychological basis of insight in first-episode schizophrenia: a pilot metacognitive study. Schizophr Res 2004;70:195-202.

32. Pru $\beta$ L, WiedL KH, Waldorf M. Stigma as a predictor of insight in schizophrenia. Psychiatry Res 2012;198: 187-193.

33. Schennach-Wolff R, Obermeier M, Seemüller F et al. Evaluating depressive symptoms and their impact on outcome in schizophrenia applying the Calgary Depression Scale. Acta Psychiatr Scand 2011;123:228-238.

34. Kemp R, David A. Psychological predictors of insight and compliance in psychotic patients. $\mathrm{Br} \mathrm{J}$ Psychiatry 1996;169:444-450.

35. Pinnenborg GH, van der GaAg M, Bockting CL, van der Meer L, Aleman A. REFLEX, a social-cognitive group treatment to improve insight in schizophrenia: study protocol of a multi-center RCT. BMC Psychiatry 2011;5:161.

\section{Supporting Information}

Additional Supporting Information may be found in the online version of this article:

Table S1. Spearman rho Correlations ( $P$-value, 2 -tailed) of raw scores of neurocognition, social cognition, clinical symptoms, and other variables with insight change in patients with psychotic disorders $(n=154)$. 